\title{
Finite Element Simulation of Multi-pass Welding Process with Rezoning Technique*
}

\author{
by Hui HUANG**,Yao ZHAO*** and Hua YUAN**
}

\begin{abstract}
Finite element simulation of welding process has been wildly employed in engineering where welding deformation and residual stress are considered. One big problem during simulation is the severe demands of capacity of hard disk and computation time, especially for large structures with long weld length or multi-pass welding. To minimize the number of unknowns in finite element model, a rezoning technique is developed to simulate the multi-pass welding process. The local nonlinear zone around the current pass is modeled with a dense finite element mesh, while the region of other passes and nearby is modeled with a coarser mesh. Then the model is redefined layer by layer to represent the filling of the welding pass and the motion of the weld arc. The rezoning procedure is implemented using a reverse mapping algorithm with Newton method to solve the high order simultaneous equations. Three-dimensional finite element simulations with and without rezoning technique are performed to obtain transient temperature, welding deformation and residual stress. By comparing the results of the model, computational efficiency and accuracy of the proposed method is confirmed.
\end{abstract}

Key Words: Finite element simulation, Multi-pass welding, Rezoning technique, Computational efficiency

\section{Introduction}

Welding technology is widely used in shipbuilding, aerospace industry, and bridge engineering for its excellent joining performance. One main problem in assembling is welding distortion which results in misalignment between assembly parts and increases fabrication costs. Furthermore, residual stress due to welding could affect structural reliability and local strength. Improper welding methods could cause brittle fracture, buckling and welding cracks, which are difficult to measure or observe during welding. Therefore, it is essential to develop an effective and efficient analysis method to estimate welding distortion and residual stress in welding structures.

In the past decades, the thermal elastic-plastic finite element method has been widely employed in simulation of welding deformation and residual stress for T-joint and butt welded plates. Also, some models have been proposed to predict welding distortion or residual stress for multi-pass welding process. Ueda ${ }^{1)}$ developed a thermal elastic-plastic FEM based on triangular element, and the welding stresses and strains in multi-pass welded butt joints of $150 \mathrm{~mm}$ thick plate were investigated. Duranton $^{2)}$ proposed an adaptive meshing technique with constraint elements to decrease the computation costs when keeping good confidence in the results. Deng ${ }^{3)}$ used a 2D axisymmetric model to predict welding stress in multi-pass butt-welded steel pipes, considering the influence of solid-state phase transformation.

Compared with 2D finite element model, a 3D finite element

\footnotetext{
*Received: 2010.11.12

${ }^{* *}$ Graduate, Huazhong University of Science and Technology

${ }^{* * *}$ Professor, Huazhong University of Science and Technology
}

model and a moving heat source can more accurately simulate the welding problems. However, a complete three dimensional thermal elastic-plastic analysis of multi-pass welding process is usually difficult to accomplish because of the demanding computational effort. The model using the usual FEM would need very fine mesh in the heat affected zone. It is necessary to reduce the unknowns of the finite element model according to the character of the welded zone.

In the present study, a rezoning technique in three dimensional FEM analysis of multi-pass welding process is developed. Welding deformation and residual stress of a plate made with low-temperature high-strength steel under multiple thermal cycles of multi-pass welding are investigated. The model is also simulated by the ordinary finite element method. By comparing the results of transient temperature, welding deformation, residual stress and computational time, effectiveness and efficiency of the proposed method is confirmed.

\section{Rezoning Technique}

Due to the characteristic of welding problems, region in the heat affected zone of the weld piece is always in highly nonlinear state while most of the domain excluding the region is still in weakly nonlinear or even elastic state. Therefore, a dense mesh in the region and a coarse mesh in the domain can be adopted in thermal mechanical analysis to minimize degree of freedoms of the model. Then the region can be redefined according to the motion of the heat source, which will improve efficiency and ensure good accuracy in solution. Lindgren proposed automatic rezoning procedure in simulation of welding based on a graded 
hexahedral element ${ }^{4)}$. Brown developed rezoning and dynamic substructuring technique to perform finite element analysis on laser welding of plate with shell element ${ }^{5}$.

There are two procedures in rezoning process. First, the coordinates of the new mesh are obtained from the old one by calculating the normalized coordinates and substituting into element shape function. Second, the solution variables are mapped from the old mesh to the new one.

\subsection{Nodes relocation}

Generally, the occupied space of new mesh should be the same as that of the old mesh, and exact location of each node in the new mesh are determined through normalized coordinates (Fig. 1). We use the method proposed by Murti and Valliappan ${ }^{6}$ ) to detect if the node is inside or outside an element. After the match relationship between the node in new mesh and the element in the old mesh is obtained, the normalized coordinates can be determined directly by a set of interpolation function for iso-parametric element. Suppose that a node $P$ locates in a known element, and the interpolation function is shown as:
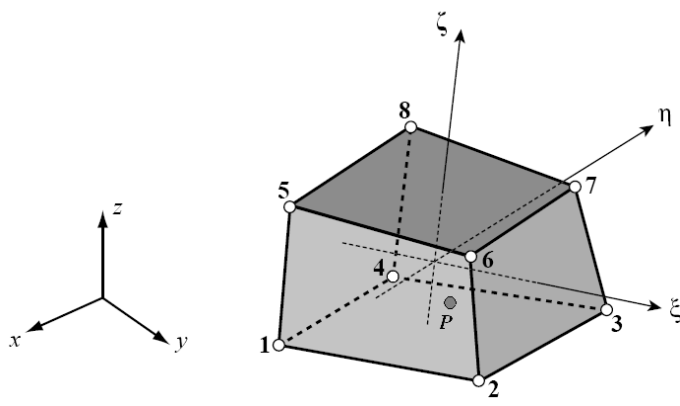

Fig. 1 A point in a hexahedral element and coordinate systems

$$
N_{i}=\frac{1}{8}\left(1+\xi_{i} \xi\right)\left(1+\eta_{i} \eta\right)\left(1+\zeta_{i} \zeta\right)
$$

The coordinate of node $\mathrm{P}$ can be transformed from normalized coordinate by the following formulas:

$$
\begin{aligned}
& x=\sum_{i=1}^{8} N_{i}(\xi, \eta, \zeta) x_{i} \\
& y=\sum_{i=1}^{8} N_{i}(\xi, \eta, \quad \zeta) y_{i} \\
& z=\sum_{i=1}^{8} N_{i}\left(\begin{array}{ll}
\xi & \eta
\end{array}\right) z_{i}
\end{aligned}
$$

As the nodal coordinates are known, the above formulas can be rewritten as [5]:

$$
\begin{aligned}
& a_{0}+a_{1} \xi+a_{2} \eta+a_{3} \zeta+a_{4} \xi \eta+a_{5} \eta \zeta+a_{6} \xi \zeta+a_{7} \xi \eta \zeta=0 \\
& b_{0}+b_{1} \xi+b_{2} \eta+b_{3} \zeta+b_{4} \xi \eta+b_{5} \eta \zeta+b_{6} \xi \zeta+b_{7} \xi \eta \zeta=0 \\
& c_{0}+c_{1} \xi+c_{2} \eta+c_{3} \zeta+c_{4} \xi \eta+c_{5} \eta \zeta+c_{6} \xi \zeta+c_{7} \xi \eta \zeta=0
\end{aligned}
$$

Here, $a_{i}, b_{i}, c_{i}$ are functions of nodal coordinates $\left(x_{i}, y_{i}, z_{i}\right)$, thus
Eq. 3 are high order simultaneous equations of $\xi$, $\eta$, and $\zeta$. For each node of the new mesh, Eq. 3 is needed to solve normalized coordinate. The authors employ Newton-Raphson method to find the approximate solution, and only a few iterations are sufficient for convergence. Finally, the coordinate of node $\mathrm{P}$ can be obtained by interpolating the corresponding normalized coordinates through Eq.2.

\subsection{Solution Mapping}

During the map solution procedure, the solution variables at the integration points of the old mesh are extrapolated to nodes of each element, and these values are averaged over all similar elements abutting each node. According to the coordinate of each integration point in the new mesh, the element of the old mesh in which the point lies is found. The location of each integration point is obtained with respect to the old mesh, and then the variables are interpolated from the nodes of the old element to the integration points of the new element. The solution continues with the mapping variables as the initial conditions.

\section{FEM simulation of multi-pass welded butt joint}

In this section, 3-D FEM analysis of multi-pass welded butt joint with rezoning technique is performed. The model used in this study is butt welded plate with length of $300 \mathrm{~mm}$, width $500 \mathrm{~mm}$ and thickness of $30 \mathrm{~mm}$. Figure 2 shows the dimension of the welded butt joint, sequence of weld passes and detail of the groove. The material of the plate is EH36, which is a type of widely used steel in shipbuilding because of its excellent low-temperature high-strength performance. All of the nine passes are performed by gas metal welding with $\mathrm{H} 08 \mathrm{Mn} 2 \mathrm{E}$ as weld filler. The welding condition of the model is given in Table 1.

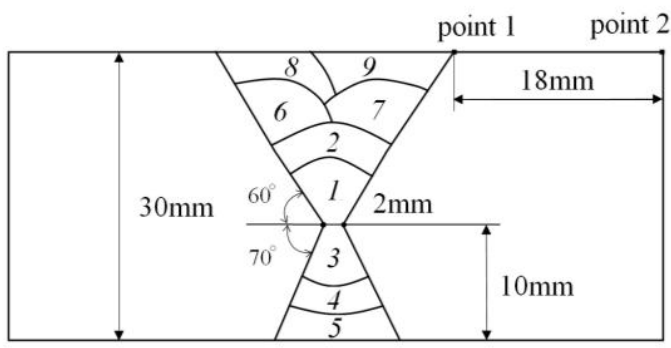

Fig. 2 Shape and size of the multi-pass welding joint

Numerical simulation of welding distortions and residual stresses needs to accurately take into account the interactions between heat transfer, mechanical fields and metallurgical transformations. In the present study, the phenomena such as arc, base metal/filler metal interactions, metallurgical transformation as well as fluid dynamics in the weld pool are not considered. 
In this study, the sequentially coupled thermal-mechanical FEM simulation based on Abaqus code is employed. First, heat transfer analysis is implemented to obtain temperature history. Secondly, mechanical analysis is performed by importing the transient temperature as thermal load.
As the stress-strain effect on thermal analysis is neglected in the sequentially coupled thermal mechanical analysis, the same finite element model in heat transfer analysis is employed here in mechanical analysis, except for the element type and boundary conditions. The applied boundary conditions in mechanical model

Table1 Welding conditions of multi-pass butt joint

\begin{tabular}{cccccc}
\hline $\begin{array}{c}\text { Pass } \\
\text { Number }\end{array}$ & $\begin{array}{c}\text { Current } \\
(\mathrm{A})\end{array}$ & $\begin{array}{c}\text { Voltage } \\
(\mathrm{V})\end{array}$ & $\begin{array}{c}\text { Speed } \\
(\mathrm{mm} / \mathrm{min})\end{array}$ & $\begin{array}{c}\text { Heat input } \\
(\mathrm{KJ} / \mathrm{mm})\end{array}$ & $\begin{array}{c}\text { Preheating/Inter-pas } \\
\text { s temperature }\left({ }^{\circ} \mathrm{O}\right)\end{array}$ \\
\hline 1 & 220 & 26 & 270 & 1.017 & 200 \\
2 & 220 & 26 & 252 & 1.090 & $160 \sim 200$ \\
3 & 220 & 26 & 318 & 0.863 & $160 \sim 200$ \\
4 & 250 & 28 & 330 & 1.018 & $160 \sim 200$ \\
5 & 250 & 28 & 282 & 1.191 & $160 \sim 200$ \\
6 & 250 & 28 & 318 & 1.057 & $160 \sim 200$ \\
7 & 250 & 28 & 276 & 1.217 & $160 \sim 200$ \\
8 & 250 & 28 & 300 & 1.120 & $160 \sim 200$ \\
9 & 250 & 28 & 324 & 1.037 & $160 \sim 200$ \\
\hline
\end{tabular}

\subsection{Heat transfer analysis}

Transient temperature filed during the welding process can be obtained by the heat transfer analysis. The material properties of weld metal are assumed to be the same with that of base metal. According to the feature of gas metal arc welding, a combined surface and body flux heat source is adopted to simulate the heat of the arc and the molten metal droplets. The arc efficiency of the welding is assumed to be $0.80 .40 \%$ of the total heat input $Q_{T}$ is given by surface flux and $60 \%$ by body flux. In this study, uniform flux distribution of both surface flux and body flux is assumed. Heat loss due to radiation and convection are considered together with a constant film coefficient of 30 $\mathrm{W} / \mathrm{m}^{2} / \mathrm{s} /{ }^{\circ} \mathrm{C}$. Latent heat is assumed to be $270 \mathrm{~J} / \mathrm{g}$, with solidus and liquidus temperature $1465^{\circ} \mathrm{C}$ and $1545^{\circ} \mathrm{C}$, respectively. User subroutine DFLUX is utilized to model heat input by surface and body flux, and the movement of the heat source.

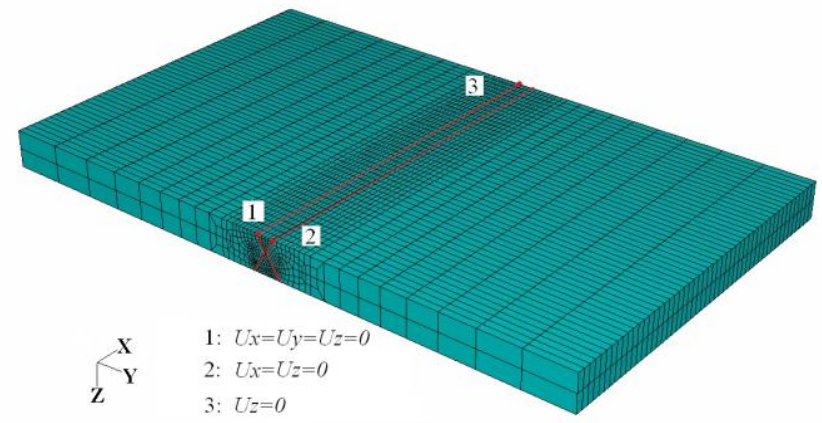

Fig.3 Boundary condition of the multi-pass welding model are shown in Fig. 3, which indicates that the restraint just prevents the rigid body motion of the weld piece during welding.

The temperature history obtained from heat transfer analysis is input as thermal load in mechanical analysis. In the present study, phase transformation induced strain and work hardening is neglected.

\subsection{FEM analysis with and without rezoning technique}

The finite element model in each welding pass with rezoning technique is shown in Fig. 4. In the rezoning model, the mesh changes after each welding pass, nodes relocation and solution mapping are performed between every two consecutive passes. The mesh of the full model without rezoning is the same with that of rezoning model in the last welding pass. The model change technique which enables the filling metal to activate/deactivate is adopted in the full model. Mesh division on the cross section of filling metal in each pass is the same in the two models, and element lengths in longitudinal direction are both $6 \mathrm{~mm}$. The node and element numbers of the first rezoning model are 8109 and 5950. The number of nodes is 19125, and that of elements is 16650 in the full model.
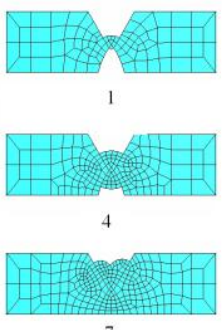
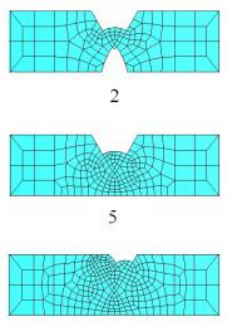
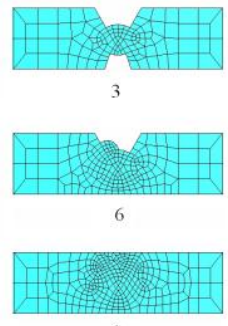

Fig. 4 Mesh of the rezoning model in each welding pass 
Procedure of the thermal mechanical analysis with rezoning technique is shown in Table 2. The steps are carried out in a shell program which executes finite element analysis and user subroutines. The welding conditions and boundary restraint of rezoning model are the same with full model.

Table2 Main steps of FEM analysis in rezoning model

Step1 heat transfer analysis with the $1^{\text {st }}$ mesh

Step2 mechanical analysis with the $1^{\text {st }}$ mesh; $i=1$

Step3 temperature field mapping from $i$ th mesh to $(i+1)$ th mesh

Step4 heat transfer analysis with the $(i+1)$ th mesh

Step5 node coordinates relocation in $(i+1)$ th mesh to occupy the same space in $i$ th mesh

Step6 solution field mapping from $i$ th mesh to $(i+1)$ th mesh

Step7 mechanical analysis with the $(i+1)$ th mesh

Step8 go to the next welding pass simulation (Step 3); $\mathrm{i}=\mathrm{i}+1$

\section{Simulated results and discussion}

\subsection{Temperature history}

Generally, mechanical behavior and phase transformation are closely related to temperature during welding, thus temperature history is significant to calculate welding deformation and residual stress. Figure 5 shows temperature history at two specific points in the full model and rezoning model. From this figure, it can be observed that the results of the models with and with rezoning technique agree well for the two points in transient time. Temperature on point 1 is higher than that on point 2 at any time since that the distance from the former to weld centerline is much closer than the latter one.

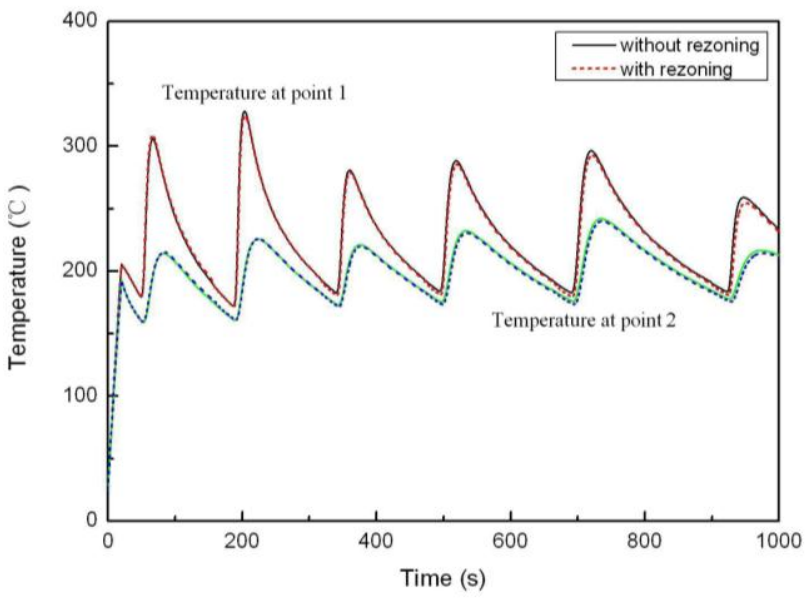

Fig. 5 Temperature history of two points

\subsection{Welding distortions}

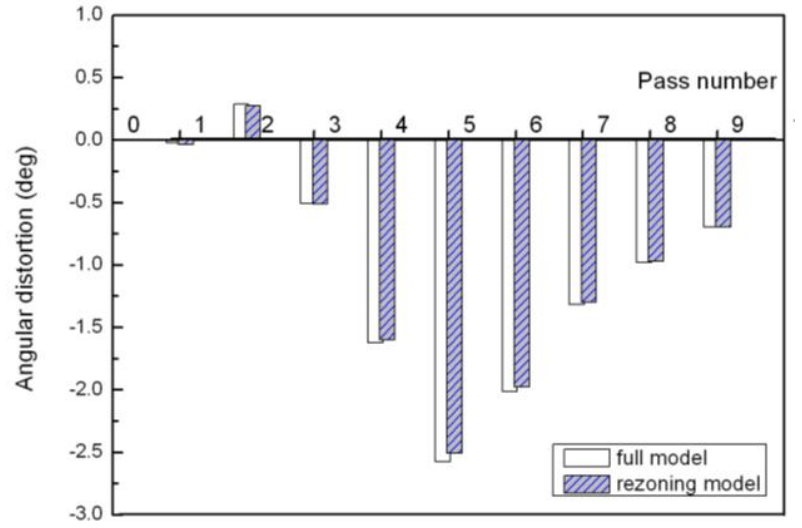

Fig. 6 Angular distortion in each welding pass

Angular distortion at the middle cross section of the plate normal to the weld line after each welding pass is shown in Fig. 6. Here, angular distortion is defined as the total changes of the angle between plate on the left side and plate on the right side. It is clear that the angular distortion depends on the location of the welding pass. If the welding pass is beneath the center of the existed fusion zone, angular distortion will reduce; otherwise, it will grow.

Transverse shrinkage and angular distortion of the two different models after cooling are shown in Fig. 7. It can be seen that the distributions of welding deformations are quite uniform along the weld line. Simulated results of rezoning model agree very well with the full model.

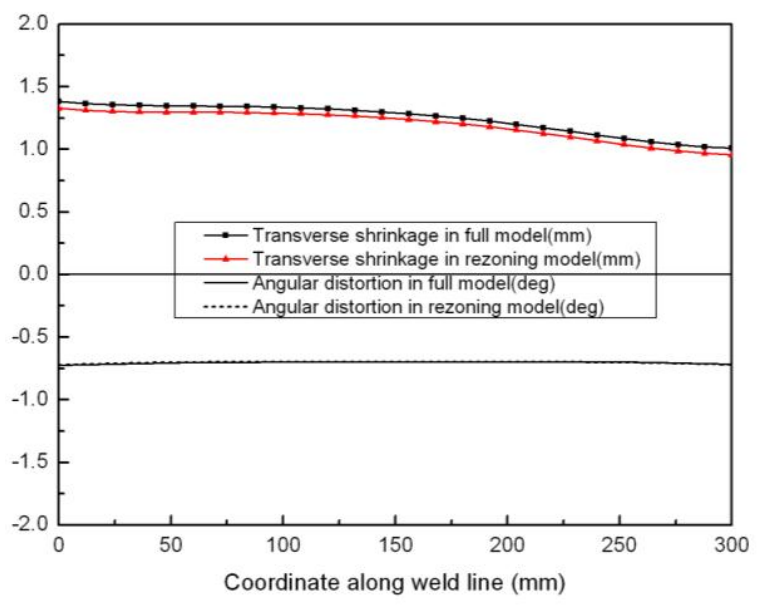

Fig. 7 Welding deformation of the multi-pass welding joint

\subsection{Residual stresses}

Figure 8 shows Mises stress field on middle cross-section of the model with and without rezoning technique. The material in the fusion zone exhibits high stress level and most of the points have reached the initial yield strength. The difference of residual stress distributions between full model and rezoning model is very small. 

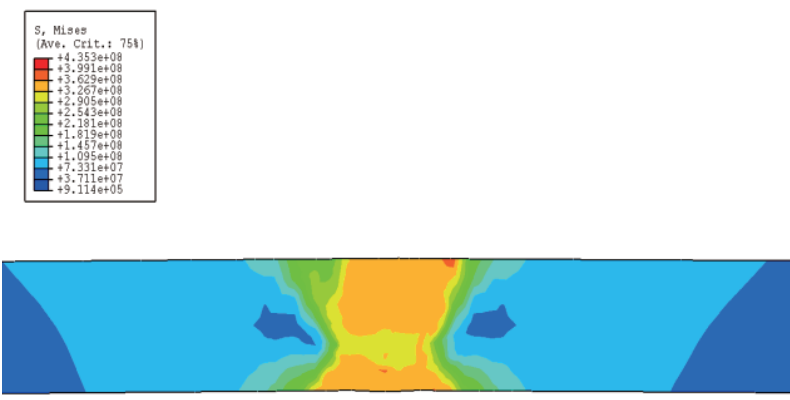

(a)
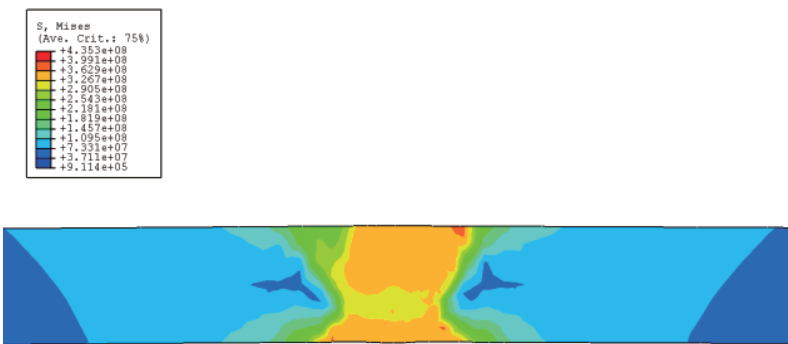

(b)

Fig.8 Comparison of Mises stress field on middle cross-section: (a) without rezoning (b) with rezoning

\subsection{Simulation time}

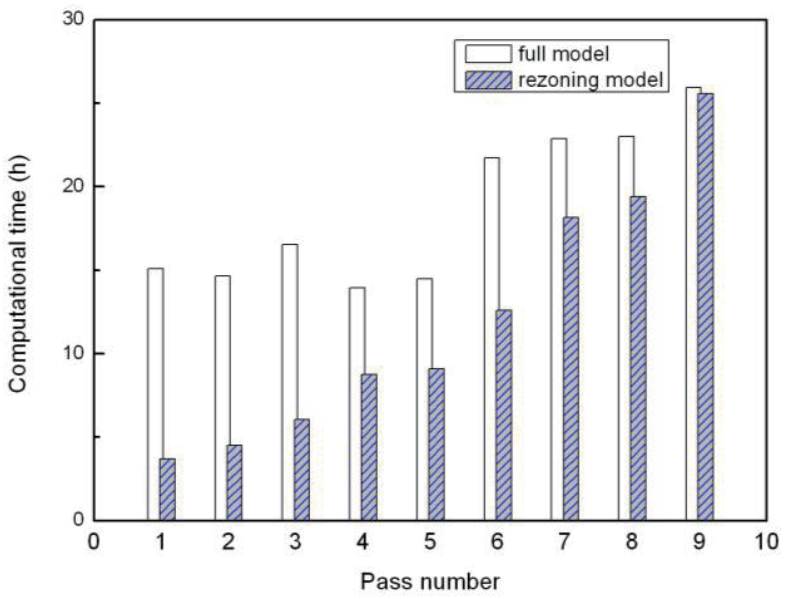

Fig. 9 Comparison of simulation time for each welding pass

Computational time for each welding pass is shown in Fig. 9. It can be found that computational time increases by pass in the rezoning model. This can be explained by the fact that the mesh scale becomes larger as the pass number increases. Total simulation time of the model with and without rezoning is $107.8 \mathrm{~h}$ and $168.3 \mathrm{~h}$, respectively.

In the present study, mesh coarsening has not been implemented. The relationship between coarsening criterion and solution accuracy is under investigation to gain more reduction in solution time.

\section{Conclusions}

In this paper, a rezoning technique for three-dimensional thermal mechanical analysis is developed to simulate the multi-pass welding process. According to the calculated results of the model with and without rezoning technique, the following conclusions can be drawn:

(1)Transient temperature, welding deformation and residual stress calculated in rezoning model agree very well with that of full model.

(2)Computational time of the simulation is reduced by $36 \%$ with rezoning technique for the studied model. Effectiveness and efficiency of the proposed method is confirmed.

\section{Reference}

1) Ueda Yukio, Eiji Takahashi, Keiji Fukuda, Keiji Nakacho: Transient and residual stresses in multi-pass welded butt joints of $15 \mathrm{~cm}$ thick plate, J. Jpn. Weld. Soc., 44-5(1974), 466-474

2) P. Duranton, J. Devauxa, V. Robin, P. Gilles, J.M. Bergheau: 3D modelling of multipass welding of a $316 \mathrm{~L}$ stainless steel pipe, J. Mater. Process. Technol., 153-154(2004), 457-463

3) D. Deng, H. Murakawa: Numerical simulation of temperature field and residual stress in multi-pass welds in stainless steel pipe and comparison with experimental measurements, Comput. Mater. Sci., 37-3 (2006), 269-277.

4) L. E. Lindgren, H. A .Haggblad, J. M. J. McDillb, a.s.oddy: Automatic remeshing for three-dimensional finite element simulation Of welding, Comput. Methods Appl. Mech. Engrg., 147-3 (1997), 401-409.

5) S. B. Brown, H. Song: Finite Element Simulation of Welding of Large Structures, ASME J. Engrg. Ind., 114(1992), 441-451.

6) V. Murti, S. Valliappan: Numerical Inverse Isoparametric Mapping in Remeshing and Nodal Quantity Contouring, Comput. Struct., 22-6(1986), 1011-1021 